EP-5

\title{
Hepatectomy outcomes in patients with hepatitis C virus-related hepatocellular carcinoma with or without cirrhosis
}

\author{
Young Ju OH, Sunghae PARK, Sang Oh YUN, Manuel LIM, Jaehun YANG, Eun Sung JEONG, Ji Eun KWON, \\ Kyeong Deok KIM, Jinsoo RHU, Gyu-Seong CHOI, Jong Man KIM*, Jae-Won JOH \\ Department of Surgery, Samsung Medical Center, Sungkyunkwan University School of Medicine, Seoul, Korea
}

Introduction: Although hepatocellular carcinoma (HCC) is rare in hepatitis C virus (HCV) patients without cirrhosis, little is known about the postoperative results of these patients. We performed this study to compare the outcomes of cirrhotic and non-cirrhotic HCV patients with solitary treatment-naïve HCC and to assess ability of non-invasive markers to predict prognosis in these patients.

Methods: Two hundred seven adult hepatectomy patients with HCV-related HCC were prospectively identified at our institution between July 2005 and May 2019.

Results: One hundred sixteen (56\%) hepatectomy patients had HCC in a non-cirrhotic liver. The non-cirrhotic group had better liver function than the cirrhotic group. Consistently, the FIB-4 grade, ALBI grade, and APRI were higher in the cirrhotic group than in the non-cirrhotic group. The cumulative disease-free survival rates and patient survival rates in the non-cirrhotic group were significantly better than in the cirrhotic group. HCC recurrence is related to major liver resection (LR) and alpha-fetoprotein (AFP) $>40 \mathrm{ng} / \mathrm{mL}$, death is related to long hospitalization, and AFP > 40 in multivariate analysis. APRI was the only predisposing factor for HCC recurrence in non-cirrhotic patients in multivariate analysis. The FIB-4 grade, ALBI grade, and the presence of cirrhosis were not related to HCC recurrence or patient survival in multivariate analyses.

Conclusions: The non-cirrhotic group had a higher survival than the cirrhotic group after curative LR, although the difference was not significant in multivariate analysis. The factors influencing HCC recurrence and patient survival were different in the cirrhotic and non-cirrhotic groups. 